\title{
Psychiatrist Health Human Resource Planning - An Essential Component of a Hospital-Based Mental Healthcare System Transformation
}

Sarah Jarmain

\begin{abstract}
The World Health Organization (WHO) defines health human resource planning as "the process of estimating the number of persons and the kinds of knowledge, skills, and attitudes they need to achieve predetermined health targets and ultimately health status objectives" (OHA 2015). Health human resource planning is a critical component of successful organizational and system transformation, and yet little has been written on how to do this for physicians at the local level. This paper will outline a framework for developing and managing key aspects of physician human resource planning related to both the quantity and quality of work within a hospital setting. Using the example of a complex multiphase hospital-based mental health transformation that involved both the reduction and divestment of beds and services, we will outline how we managed the physician human resource aspects to establish the number of psychiatrists needed and the desired attributes of those psychiatrists, and how we helped an existing workforce transition to meet the new expectations. The paper will describe a process for strategically aligning the selection and management of physicians to meet organizational vision and mandate.
\end{abstract}

\section{Introduction}

Health human resource planning is a critical component of successful organizational and system transformation. It is increasingly recognized that robust human resource manage- ment practices are a necessary condition for the successful reform of healthcare services (Bach 2001), and these practices need to be developed in conjunction with the desired reforms. To be effective, physician human resource planning should involve consideration of the number of physicians required, the types of roles they need to fulfill, the competencies to fulfill these roles and the attributes of the individual physicians that will support the delivery of evidence-based, high quality, patient-centred care. While there are many frameworks that outline the stages of strategic human resource planning and talent management, they can be summarized as follows:

1. Planning - identify the organization's human resource needs as determined by a review of organizational need, strategic direction and current and future gaps in numbers or competencies

2. Selection - sourcing, recruiting and selecting individuals with the right knowledge, skills and attitudes

3. Alignment - developing an onboarding process that introduces the candidate to the desired organizational behaviours and performance objectives; subsequent performance development and management to align with organization vision and values

4. Retention - ongoing engagement and empowerment, learning and development, rewards and recognition

5. Succession - career development, transition management and succession planning 
This paper will review the relevant literature in physician health human resource planning and describe how one organization (St. Joseph's Health Care London) developed processes to support the planning, selection and alignment of physicians through a period of significant organizational change.

\section{Background}

The Mental Health Care Program, part of St. Joseph's Health Care London, is a mid-sized academic mental health program based on two sites that serves a catchment area of approximately 1.5 million people in south western Ontario. It consists of 89 forensic psychiatry beds and 153 non-forensic specialized beds serving individuals from age 14 and up with a range of complex psychiatric disorders. However, it has undergone considerable change over the last 15 years.

In 1997, the Health Services Restructuring Commission (HSRC) in Ontario issued a number of recommendations related to hospital restructuring, including direction for the existing provincial psychiatric hospitals to be divested from the Ontario government and for investment to be made in building new mental health facilities across the province. In the southwest region, these directives resulted in the following:

- 2001: Divestment of London Psychiatric Hospital and St. Thomas Psychiatric Hospital to St. Joseph's Health Care London

- 2006-2007: Restructuring of beds and services to diagnostically based groupings to better align with peer service providers, academic mandate and best practice

- 2010, 2011, 2013, 2014: Divestment of a total of 138 inpatient beds and resources with associated outpatient services to four receiving hospitals

- 2010, 2011, 2014: Reduction in inpatient beds in three phases

- 2013, 2014: Construction of two new facilities for the mental healthcare program and services at St. Joseph's Health Care London - Southwest Centre for Forensic Mental Health Care (completed 2013) and Parkwood Institute Mental Health Care Building (completed 2014)

These structural changes within the region's specialized mental health delivery system required transformational changes of the medical staff in both the number of physicians and their competencies and characteristics. These changes included elimination of psychiatrist positions, transfer of psychiatrists with the divestment of beds and services to other communities and a requirement for increased academic focus and greater specialization. There was a corresponding shift in the philosophy of care from a more traditional, custodial approach to a care model consistent with the principles of recovery and rehabilitation, which similarly required different roles, skills and attitudes within the psychiatric workforce providing care.

While these structural and cultural transformative changes impacted significantly on the human resource needs of all disciplines, it was particularly essential to attend to these issues within the medical staff, for the following reasons. Whether they hold a position of authority or not, physicians are often looked to as informal leaders by the rest of the interdisciplinary team, and therefore often have a significant impact on the culture of the team. They are also a relatively scarce resource. Lau and colleagues outline a number of studies that suggest that Canada is already experiencing a shortage of psychiatrists and that this problem is projected to worsen significantly over the next 10 years (Lau et al. 2015). Given this shortage, and the fact that it can take from 6 to 18 months from recruitment through to a physician starting with an organization, the need to retain and develop our existing workforce of psychiatrists to be able to continue to serve our region was felt to be essential.

\section{Planning - Determination of Physician Numbers}

Traditional approaches to physician health human resource planning have focused on the number of physicians required in a particular region (typically number of physicians per 100,000 population). However, much has been written on the limited utility of this approach because it fails to factor in a variety of contextual factors, including those related to the provider (e.g., stage of career, gender, productivity, degree of subspecialization), the geography (e.g., urban versus rural) and the population being served (e.g., socioeconomic factors, disease burden) (Fooks et al. 2002). Furthermore, this approach has limited utility when applied to the level of the individual organization. Sargeant and colleagues, in their position paper, "Psychiatric Human Resources Planning in Canada," suggest that the judicious application of several approaches, including benchmark, needs-based and demand-based principles, may be more useful than any one alone, as they are suited to different levels of analysis (Sargeant et al. 2010).

In the St. Joseph's Mental Health Care transformation, there was a need to determine the physician full-time equivalents (FTEs) required to support the clinical and academic mandate of the organization through the restructuring process. By identifying the desired service capacity (e.g., number of beds, outpatients served) and relevant workload variables (e.g., patient complexity, desired length of stay, assistance from team members) at each stage of the restructuring, a model was created using previously established workload benchmarks that identified the FTE of physicians needed per service area for each stage. This method allowed for proactive planning to determine the number of physician positions to transfer with divested resources or the number to be reduced to align with the proposed bed reductions. It also provided an opportunity to 
explore innovative solutions to retain physicians in the region, for example, partnering with community agencies that needed to recruit psychiatrists to meet their increasing needs.

While estimating the numbers of physicians required and the associated workload is an important underpinning of human resource planning, the area has evolved to include discussions around strategic talent management and high-performance work systems - "a group of separate, but interconnected human resource practices that together recruit, select, develop, motivate and retain employees" (Zacharatos et al. 2005). There is growing evidence that healthcare organizations that have implemented and sustained such evidence-based approaches have documented substantial improvements in operating margins, workforce engagement and performance, faculty promotion rates, and quality of care (Leggat et al. 2011; Fox et al. 2011). A fundamental aspect of these approaches has been to identify the desired behaviours and competencies that will support both the strategic direction of the organization and the desired culture.

There has been a growing recognition that the environment in which physicians practice today is significantly different from that of the past. Recent healthcare reform efforts in the United States and Canada have focused on the "triple aim" of improving healthcare for individuals, improving population health and lowering costs. Physicians, who traditionally have practised with considerable autonomy, are now required to become members of the team-based patient care models that are necessary to support these aims (Mahon et al. 2013; Halpern et al. 2001). The Royal College of Physicians and Surgeons of Canada has recognized this change through its work with the CanMEDS framework, which is in the process of being updated (Frank et al. 2014). In addition to the role of medical expert, physicians are described as having the roles of communicator, collaborator, leader, health advocate and scholar. Updates from prior versions have included an increased focus on quality and patient safety, patient as partner and the role of health information technology.

\section{Development of Physician Human Resource Planning Principles and Selection Criteria}

Anticipating the significant impact of the HSRC directives on hospital restructuring, and in turn on physicians and dentists, a guiding document was prepared in 1997 by representatives from the London Hospitals Medical/Dental Staff Organizations, providing a "principled process" to guide medical human resource planning (London Hospitals Task Force on Medical Human Resources, 1997). The intent was to develop plans consistent with "the mission, role and strategic direction for each institution while considering the impact on patient care, academic and research missions." It formed the basis for the development of a subsequent document in 2010 titled "Human Resource Planning for the Evolving Specialized Mental Health and Forensic Facilities at RMHC/SJHC.” (St. Joseph's Health
TABLE 1.

Guiding Principles and Physician Selection Criteria

\section{Guiding Principles}

- Medical/dental resource planning and decision-making will be fair and transparent.

- All physicians/dentists will have equal opportunity to apply for positions as these become available.

- Hospital, academic (teaching and research) and community needs will be considered in decision-making, together with physician skill and time allotment.

- All stakeholders (particularly administrative partners within the hospital but including other departments/programs) will be consulted in the process

- The best person for the position will be selected irrespective of prior hospital attachment or contractual relationship.

- An appeals process will be identified.

\section{Physician Selection Criteria}

- Ability and qualifications to provide evidence-based psychiatric care both in a broad, generalist context and in a range of subspecialty areas, including recovery models of care, evidence-based psychotherapies, psychopharmacology and electroconvulsive therapy (ECT)

- Ability for specialized physicians to upgrade where needed, within a reasonable amount of time

- Willingness to provide both inpatient and outpatient services

- Collegiality (i.e., the ability to work in a professional manner with physician colleagues and other hospital staff)

- Contribution (i.e., the degree to which the physician contributes to the program, hospital, university and community)

- Physician utilization of resources (e.g., case volumes, lengths of stay, number of separations) are within normative levels

- Physicians will be familiar with CanMEDs roles and will practise in this context

- Any complaints and concerns expressed by staff/patients regarding the physician that have led to performance management processes may factor into selection process

- Strong preference will be given to full-time clinical academics with an adjunct appointment being a minimal requirement

- For areas of Royal College subspecialization (child and adolescent, geriatric, and forensic psychiatry), demonstration of defined competencies and affiliations in those specialties will be required

- Seniority will not be a consideration unless all other factors between two potential candidates are judged to be equal; in this case, seniority may be the final deciding criterion 
Care London Department of Psychiatry, 2010). The physician leaders within the mental healthcare program developed this document with input from key stakeholders, including the medical staff, operational program leaders and the vice-president, medical. The document identified principles and processes and the factors to be considered for physician selection. It also outlined key issues to be considered in the work, such as anticipated decreases in program funding, the need for accessible and effective ambulatory services in the region to support the reduced number of beds, the need for greater collaboration with community partners, changing demographics with an aging population, and the shift in the model of care to a recovery philosophy and emphasis on psychiatric rehabilitation. Table 1 outlines the specific guiding principles and selection criteria that were used.

The above selection criteria were translated into a "physician selection scorecard," whereby the selection criteria were weighted based on importance to the strategic direction of the organization, and each physician was rated on each of the criteria through a consensus process. Based on these ratings, it was determined whether a physician was a high performer, a solid performer or a low performer and therefore needing improvement. The physicians were provided feedback on where they were perceived to contribute strength to the organization and where they needed improvement. Based on this feedback, a performance development plan was collaboratively created, with targets and timelines for improvement. In some situations, it was clear that the physician would not be able to meet the future requirements of the position (e.g., would not be eligible for an academic appointment with the university) and in this case discussion centred around transition planning options such as retirement or alternative work within the region.

\section{Results and Next Steps}

In 2007, St. Joseph's Mental Health Care program had a total allocation of 64.3 FTE physicians and was staffed with 60 individuals, of which 34 (or 57\%) were full-time clinical academics. Through the restructuring process, 10 individuals followed the divested beds and resources, and 5 individuals developed retirement/transition plans to leave the organization. Three physicians undertook part-time work in the community, providing collaborative care to community mental health and addiction agencies. As of 2015, the program's physician allocation was 42.5 FTEs, with a head count of 45 . Of those physicians, 35 (or 78\%) were full-time clinical academics, six were adjunct faculty, and four were hospitalists providing medical care. From a qualitative standpoint, the sense is that physician engagement has significantly improved, with increased participation in program planning, medical staff meetings and academic deliverables. Workload remains a challenge due to higher-than-predicted occupancy rates and shorter lengths of stay, but there is an equitable and transparent process to deal with these issues and plan for future changes in care delivery.

In regard to next steps, the organization is embarking on an initiative to further improve physician engagement through a focus on shared leadership practices, increased emphasis on performance measurement and ongoing quality and process improvement. Discussions are underway with a peer tertiary mental healthcare program to share elements of the physician human resource planning framework and evaluate the spreadability of the approach. There is a need to evaluate these approaches in a more formalized way and look at how they might be adapted for different settings and contexts. HQ

\section{Lessons Learned}

- Physician engagement - A key success factor in our work was the engagement of our front-line physicians in the selection of principles to guide our transformation work. This involvement facilitated the later implementation of the principles and contributed to the development of a culture of transparency and equity.

- Communication - We initially underestimated the need for repeated and varied communication to the physicians, given the speed at which rumours and misinformation can spread. We addressed this concern by providing monthly updates at our medical staff meetings, even if there wasn't new information to share, along with the rollout of key information through our physician leaders and one-on-one meetings with the physicians. This was in addition to the monthly staff and physician forums that are co-led by the vice-president, mental health and the site chief.

- Planning approaches - A challenge in the physician planning process was the significant lead time required for making any change to physician staffing for the organization. This longer lead time was due to a combination of long notice periods for salaried physicians (approximately 12 months), the need to demonstrate a robust performance management process for those physicians who weren't meeting the future requirements of the organization, and the typical time to recruit a new psychiatrist, which could range from 6 to 12 months. In addition to these factors, it was important to have mitigation strategies in place when physicians left the organization, well before their position was reduced. We dealt with this problem by identifying who might be willing to take on additional work on a temporary basis and ensuring we compensated individuals for this increased workload. 


\section{References}

Bach, S. 2001. "HR and New Approaches to Public Sector Management: Improving HRM Capacity.” Workshop on Global Health Workforce Strategy, Annecy France, December 9-12, 2000.

Fooks, C., K. Duvalko, P. Baranek, L. Lamothe and K. Rondeau. 2002. "Health Human Resource Planning in Canada: Physician and Nursing Workforce Issues; Appendix A - Literature Review." Commission on the Future of Health Care in Canada. Retrieved November 11, 2015. <http://cprn.org/documents/14944_en.pdf>

Fox, S., S.A. Bunton and V. Dandar. 2011. The Case for Strategic Talent Management in Academic Medicine. Washington, DC: Association of American Medical Colleges.

Frank, J.R., L. Snell and J. Sherbino. 2014. The Draft CanMEDS 2015 Physician Competency Framework -- Series III. Ottawa, ON: The Royal College of Physicians and Surgeons of Canada.

Halpern, R., Lee, M.Y., Boulter, P.R., Phillips, R.R. 2001. "A Synthesis of Nine Major Reports on Physicians' Competencies for the Emerging Practice Environment." Academic Medicine 76(6):606-15.

Lau, T., D. Zamani, E.K. Lee, K.D. Asli, J. Gill, N. Brager, R. Hawa, W. Song, E. Gill, R. Fitzpatrick, N.M. Menezes, V.H. Pham, A.B. Douglass, S. Allain, G.B. Meterissian, N. Gagnon, H. Toeg and C. Murphy. 2015. "Factors Affecting Recruitment Into Psychiatry: A Canadian Experience." Acad Psychiatry 39(3): 246-52.

Leggat, S.G., T. Bartram, and P. Stanton. 2011. "High Performance Work Systems: The Gap Between Policy and Practice in Health Care Reform." J Health Organ Manag 25(3): 281-97. doi:10.1108/14777261111143536

London Hospitals Task Force on Medical Human Resources. 1997. "The Path of Development: City-Wide Medical and Dental Staff Human Resources Planning." Unpublished document. St. Joseph's Health Care London, London Canada.
Mahon, K.E., M.K. Henderson and D.G. Kirch. 2013. "Selecting Tomorrow's Physicians: The Key to the Future Health Care Workforce." Acad Med 88(12): 1806-11. doi:10.1097/ACM.0000000000000023

Ontario Hospital Association (OHA). 2015. "Physician Leadership Resource Manual.” Retrieved November 11, 2015. <https://www. oha.com/CurrentIssues/keyinitiatives/PhysicianandProfessionalIssues/ QPSGT/Pages/PhysicianLeadershipResourceManual.aspx>.

Sargeant, J. K., T. Adey, F. McGregor, P. Pearce, D. Quinn, R. Milev and N. Dada. 2010. "Psychiatric Human Resources Planning in Canada.” Canadian Journal of Psychiatry 55(9): 1-20.

St. Joseph's Health Care London - Department of Psychiatry. 2010. "Human Resource Planning for the Evolving Specialized Mental Health and Forensic Facilities at RMHC/SJHC." Unpublished document. St. Joseph's Health Care London, London Canada.

Zacharatos, A., J. Barling, and R.D. Iverson. 2005. "High-Performance Work Systems and Occupational Safety.” J Appl Psychol 90(1): 77-93. doi:10.1037/0021-9010.90.1.77

\section{About the Author}

Sarah Jarmain, MD, FRCPC, is the site chief, mental health care; chair, medical advisory committee and director, medical quality at St. Joseph's Health Care London, and an associate professor in the department of psychiatry, Western University. She has worked as a physician administrator since 2001 and has developed models for physician HR planning, workload measurement, physician engagement and leadership, and teaches widely in the areas of leadership, change and resilience. For more information, Dr. Jarmain can be reached by email at: Sarah.Jarmain@sjhc.london.on.ca. 